\title{
Performance Enhancement of CPMIMO-OFDM System using Blind Channel Estimation
}

\author{
Megha Kimothi \\ DIT UNIVERSITY \\ Dehradun 248001 India
}

\author{
Vivek Kumar Gupta \\ DIT UNIVERSITY \\ Dehradun 248001 India
}

\author{
S.C.Gupta \\ DIT UNIVERSITY \\ Dehradun 248001 India
}

\begin{abstract}
This paper deals with the blind channel estimation in $\mathrm{CP}$ MIMO-OFDM system based on subspace algorithm with reduced time samples to get time invariant system, eliminating the pilot based channel estimation and utilizing the bandwidth. This paper uses the statistical blind estimation technique by using second order statistic and in this the estimates can be obtained in a simple form by optimizing a quadratic cost function. These algorithms use the orthogonality of the noise and signal subspaces of the correlation matrix of the received signals to estimate the unknown channel coefficients. Simulation results show that the proposed approach improving the performance, observed by the graphs SER/SNR and MSE/SNR.
\end{abstract}

\section{Keywords}

Blind channel estimation, MIMO-OFDM, CPSOS, ZPSOS, mean square error, subspace algorithm.

\section{INTRODUCTION}

Channel estimation plays an important part in communication systems. It can be employed for the purpose of detecting received signal, improving the capacity of orthogonal frequency division multiple access (OFDMA) systems and improving the system performance in terms of bit error rate (BER).

Channel estimation can be performed in three ways. They are

(i)Training-based channel estimation

(ii)Blind channel estimation and

(iii)Semi blind channel estimation.

The blind channel estimation is carried out by evaluating the statistical information of the channel and certain properties of the transmitted signals. Although higher-bandwidth efficiency can be obtained in blind techniques due to the lack of training overhead, the convergence speed and estimation accuracy are significantly compromised. Blind Channel Estimation has its advantage in that it has no overhead loss, so in this paper we are using blind channel estimation. Additionally, because of the time-varying nature of the channel in wireless applications, the pilot/training sequence needs to be transmitted periodically, causing further loss of channel throughput. Blind channel estimation methods avoid the need for training sequence.

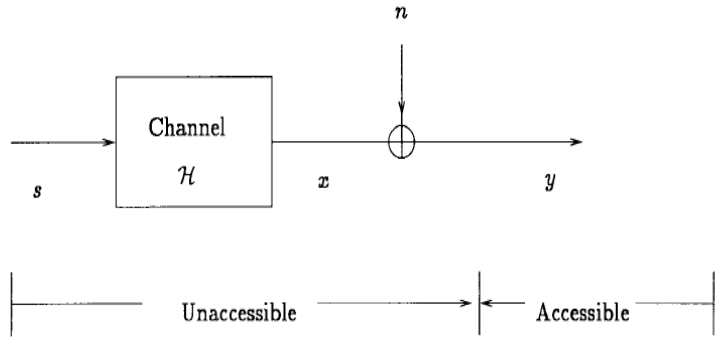

Fig 1. Schematic of blind channel estimation
Blind channel estimation plays an important role in digital communication systems. To estimate a channel, its transfer function needs to be identified either implicitly or explicitly. The difficulty of this problem starts from the fact that the input to an unknown channel (the transmitted signal) is not available at the channel output (receiver input). Hence the traditional MMSE approaches are not applicable. The solutions to the blind identification problem can be found in [1] where the essential tool is the use of higher order statistics of the channel output. On the other hand, papers [2] presented results that are based on the second order statistic. The basic blind channel estimation problem involves a channel model shown in Fig.1, where "s" input signal passing through a channel of transfer function " $H$ ", output of which is " $x$ " in which noise will be added and finally " $y$ " is obtained but in this only the observation signal is available for processing in the identification and estimation of channel. This is in contrast to the classical input-output system identification and estimation problem where both input and observation are used. Techniques for estimating the channel and the source symbols must use either a training sequence or exploit some other property of the source symbols. Training sequences, however, consume transmission bandwidth, especially when the channel is time varying and a training sequence must be sent frequently. Therefore blind techniques exploiting a known property of the source have become popular [3]. These techniques usually exploit the (second or higher order) statistics [4] to identify the channel. The statistical methods normally require many symbols to be received before the channel can be identified accurately. These methods rely on assumptions on the statistics of the input sequence means in these method the statistics of the input signal is known to us so they comes under statistical approaches while the latter method make no such assumption [5][6].These categories, i.e., deterministic methods are generally favored when the input statistics are unknown, or there may not be sufficient time samples to estimate them. It is important to point out that the above blind estimation methods are blind only in the sense that the system (channel) input is not used in the estimation; they do require some statistical assumptions on the input. However, the statistical model of the input may not be available, or there may not be enough data samples to find a reasonably accurate statistical estimate. For example, in a fast fading environment, the multipath channels in wireless communications vary rapidly, and there is only few data samples related to the same channel characteristics. In this kind of scenario, it is not feasible to assume that the estimates of the signal are close enough to their true (known) values. Hence, it may be easier to solve this problem by considering the input as a deterministic signal which has been done in [5][6]. 


\section{MIMO-OFDM}

MIMO-OFDM is one of the promising scheme for attaining high data rate and large system capacity over wireless networks. Demands for high data rate and large system capacity based wireless communication services are rapidly increasing. MIMO-OFDM allows an effective increase in data rate and capacity in a mobile wireless link without additional power or bandwidth consumption. And it also allows a notably reduction in system complexity. So activities of recent industry suggest that the use of MIMO-OFDM is particularly promising for broadband wireless access. Blind channel estimation in MIMO-OFDM systems has been a very active research area recently.

Orthogonal frequency-division multiplexing (OFDM) combined with multiple antennas at both transceiver sides has received attention over the last decade for its promising capability to combat the multipath fading and boost the system capacity [7]. The SS method has simple structure and achieves good performance, but it meets several difficulties when applied to MIMO OFDM systems [8][9]. Firstly, more receive antennas than transmit antennas are required, which hardly holds since the symmetric links play a major role in most wireless transmission standards, e.g. the $2 \times 2 \mathrm{MIMO}$ for IEEE $802.11 \mathrm{n}$ device . However, if oversampling is used at the receiver, the case $N_{R} \leq N_{T}$ is also possible [10]. Besides, equal number of the transceiver antennas is obviously used in the current SISO OFDM transmission schemes, e.g. IEEE 802.11a standard. Secondly, even for the cases with more receive antennas, the precise knowledge of the channel order must be obtained, which is very difficult in practice. The order over-estimation may produce an illconditioned channel matrix which degrades the performance of the SS method or sometimes fails the channel estimation. To solve these problems, a zero-padding (ZP) based MIMO OFDM was suggested in [12]. In this technique consecutive zeros are padded at the end of each OFDM block. This method will be referred to as ZPSOS, where SOS is used for "second order statistics". Although ZPSOS displays many advantages, a major problem that prevents its application is the incompatibility to most existing OFDM standards or the future 4G MIMO-OFDM standards [11]. Therefore we use cyclic prefix instead of zero padding as it is compatible with the previous standard as well as the 4G MIMO-OFDM. SS algorithm is developed that is suitable for CP based MIMO OFDM systems by applying an appropriate re-modulation on the received signal blocks [12]. But it is also applicable only for time invariant system, so the time averaging should be reduced in the propose method and for that time, channel remain invariant and correlation matrix can be estimated.

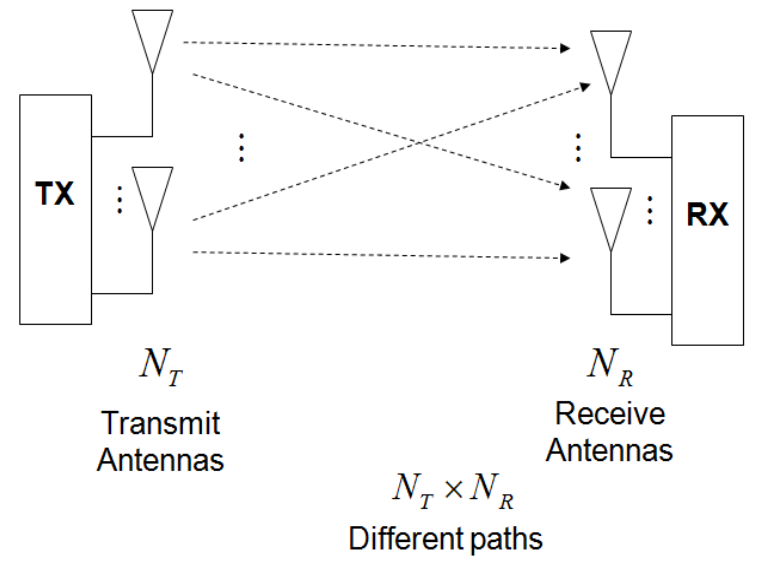

Fig.2. MIMO-OFDM
We consider $N_{T}$ transmit and $N_{R}$ receive antennas in a MIMO -OFDM system, employing $N_{C}$ sub carriers. Let $x[k] \stackrel{\text { def }}{=}$ $\left[x 1[k] x 2[k] \ldots \ldots \ldots N_{T}[k]^{T}\right]$

and $y[k] \stackrel{\text { def }}{=}\left[y 1[k] y 2[k] \ldots \ldots \ldots y N_{R}[k]^{T}\right] \quad$ where $x_{q}[k]$ and $y_{p}[k]$ are the symbol transmitted at the $q$ th transmit antenna and the symbol received at the $p$ th receive antenna respectively. Then, the input vector can be written as $\left.x \stackrel{\text { def }}{=} x[0]^{T} x[1]^{T} \ldots . . . x\left[N_{C}-1\right]^{T}\right]^{T}$ and the observation can be written as $y \stackrel{\text { def }}{=}$ $\left.y[0]^{T} y[1]^{T} \ldots \ldots y[N c-1]^{T}\right]^{T}$

Let the noise defined in a similar way represented by $\mathrm{n}$. Then, the input-output relation of the MIMO-OFDM system may be expressed by

$$
\mathrm{y}=H \mathrm{x}+\mathrm{n}
$$

where matrix $\mathrm{H} \stackrel{\text { def }}{=} \operatorname{diag}\left(\mathrm{H}[0] \cdot \cdot \cdot \mathrm{H}\left[N_{C}-1\right]\right)$ has size $\left(N_{R} N_{C}\right) \times\left(N_{T} N_{C}\right)$, with diagonal blocks defined as

$\mathrm{H}[\mathrm{K}]=\left[\begin{array}{cccc}h_{1,1}[k] & h_{1,2}[k] & \ldots & h_{1, N_{T}}[k] \\ h_{2,1}[k] & h_{2,2}[k] & \ldots & h_{1, N_{T}}[k] \\ \vdots & \vdots & \ddots & \vdots \\ h_{N_{R}, 1}[k] & h_{N_{R}, 2}[k] & \ldots & h_{N_{R}, N_{T}}[k]\end{array}\right]$

And $h_{p, q}[k]$ denotes the frequency response over the kth subcarrier from the qth transmit to the pth receive antenna. In this paper, our interest lies in the blind estimation directly from the observation $\mathrm{y}$ of the channel coefficients, i.e., $h_{p, q}[k]$.

\section{BLIND ESTIMATION BASED ON SUBSPACE}

Perhaps the most popular blind algorithm is the so called subspace (SS) based algorithm which was originally developed for single-input multiple-output (SIMO) frequency selective channels in [9]. An orthogonality property between "signal" and "noise" subspaces is exploited to build some quadratic form whose minimization yields the desired estimates up to a scale factor [13]. Second-order-statisticsbased blind approaches, subspace based estimation is attractive since estimates can often be obtained in a simple form by optimizing a quadratic cost function.

\subsection{Second Order Statistic}

By comparing the paper [14][15] with [16][17] it is observed that blind channel estimation using second-order Statistics[14][15] can potentially achieve superior performance for a given time averaging interval than approaches based on higher order statistics i.e. [16][17] .So from this statement it is clear that why second order statistics is used instead of higher order statistics in the proposed method.

\subsection{Proposed Approach}

Blind channel estimation has received a great deal of attention and has become a vital area of research. There lot of research has been done in blind channel estimation using subspace approach. I am basically working on blind channel estimation of MIMO-OFDM using subspace algorithm in which the channel matrix has been estimated as it is unknown, by time averaging process over multiple received sample but during this process the channel should be invariant, which is not possible because the channel remain invariant for the very initial and short period of time. Therefore the time averaging should be reduced by exploiting frequency correlation among 
adjacent subcarrier and estimate the channel matrix. Then applying EVD [19] to channel matrix to get eigenvector of noise subcarrier through which the quadratic cost function can be constructed and channel estimation can be done by optimizing that quadratic cost function which we can see in fig. 3. Since the quadratic cost function is constructed from the eigenvectors of the noise subspace obtained from the EVD, the accuracy of the eigenvectors obtained from the sample correlation matrix dominates the performance of the estimation.

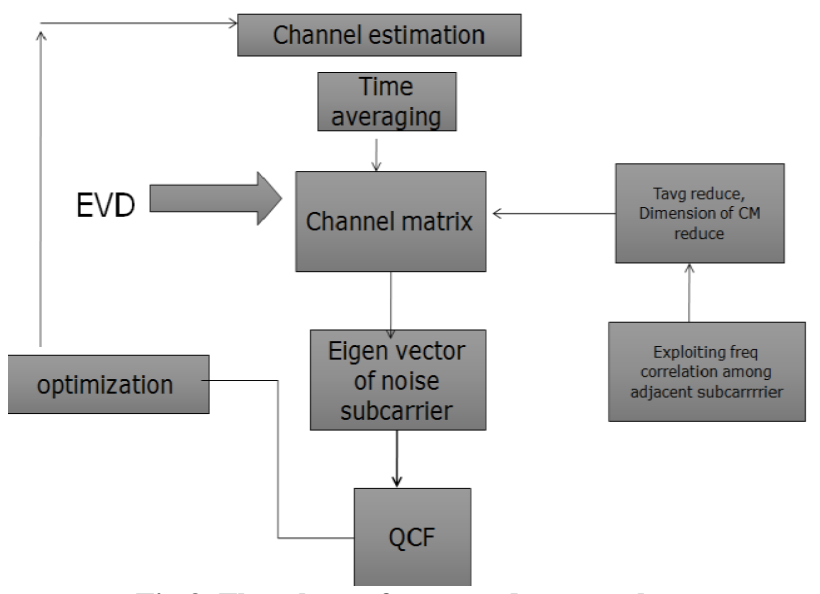

Fig.3. Flowchart of proposed approach

Let the index set of $N_{C}$ subcarriers $\Omega \stackrel{\text { def }}{=}\{0,1, \ldots, N c-1\}$, divided into $P$ disjoint subsets assuming that $\left(N_{C} / P\right)=\zeta$ with each subset denoted as $\Omega p \stackrel{\text { def }}{=}\left\{\omega_{p, 1} \omega_{p, 2} \omega_{p, \zeta}\right\}$, now equations can be rewritten for pth subset as

$$
\begin{aligned}
& x_{p}=\left[x\left[\omega_{p, 1}\right]^{T} x\left[\omega_{p, 2}\right]^{T} \ldots x\left[\omega_{p, \zeta}\right]^{T}\right]^{T} \\
& \mathrm{p}=1,2,3 \ldots \ldots \ldots . . \mathrm{P}
\end{aligned}
$$

The identification of $H p$ can then be achieved based on $R_{y_{p}}=E\left[y_{p} y_{p}^{H}\right]$, which can be rewritten as

$$
R_{y_{p}}=H_{p} R_{x_{p}} H_{p}^{H}+R_{n_{p}}
$$

Since the $P$ adjacent subcarriers are assumed to reside inside the coherence bandwidth so $\bar{H}=H_{1}=H_{2}=. \quad$. . $=H_{p}$ . Hence, an estimate of the correlation matrix in (6) can be obtained by

$\hat{R}_{\bar{y}}=\frac{1}{P T_{a v}} \sum_{J=1}^{T_{a v}} \sum_{p=1}^{P} y_{p(j)} y_{p(j)}^{H}$

Therefore, the number of the time samples $T_{a v}$ required can significantly be reduced since the dimension of the correlation matrix is reduced by a factor of $P$, when we apply EVD to $R_{y_{p}}$ , we get signal subspace denoted as $R\left(U_{S}\right)$ and the noise subspace denoted as $R\left(U_{n}\right)$, with $d_{s} \stackrel{\text { def }}{=} \operatorname{rank}(\bar{H})=N_{T} \zeta$ and $d_{n} \stackrel{\text { def }}{=}\left(N_{R}-N_{T}\right) \zeta$. The quadratic cost function given by $C(\bar{H})=\sum_{j=d_{s}+1}^{d_{s}+d_{n}}\left\|\hat{u}_{j}^{H}\right\|_{2}^{2}$ and minimizing the quadratic cost function $C^{\prime}(\bar{H})=\sum_{j=d_{s}+1}^{d_{s}+d_{n}}\left\|\bar{H}^{\prime T} \hat{u}_{j}^{*}\right\|_{F}^{2}=\operatorname{tr}\left(\bar{H}^{\prime T} \widehat{\Psi}\right)$

Where $\widehat{\boldsymbol{\Psi}} \stackrel{\text { def }}{=} \sum_{j=d_{s}+1}^{d_{s}+d_{n}} \widehat{\boldsymbol{u}}_{\boldsymbol{j}}^{*} \widehat{\boldsymbol{u}}_{\boldsymbol{j}}^{r} \boldsymbol{\epsilon} \mathrm{C}^{\left(\boldsymbol{N}_{\boldsymbol{R}} \zeta\right) \times\left(\boldsymbol{N}_{\boldsymbol{R}} \zeta\right)}$, from the RayleighRitz theorem, we know that $\gamma_{1}(\widehat{\Psi})+\ldots+\gamma_{r}(\widehat{\Psi})=$ $\min _{Q^{H} Q=I} \operatorname{tr}\left(Q^{H} \widehat{\Psi} Q\right)$

for all $Q \in \mathrm{C}^{\left(N_{R} \zeta\right) \times r}$ where $r$ is a given integer with $1 \leq r$ $\leq N_{R} \zeta$.
Main steps involves to estimate a channel

- The first step would be to compute $\hat{R}_{\bar{y}}$ which we can calculate from " (7)".

- Next step would be, apply EVD to $\hat{R}_{\bar{y}}$ then find out eigenvector $\hat{u}_{j}, \mathrm{j}=d_{s}+1-\quad-\quad-d_{s}+d_{n}$ which corresponds to $d_{s}$ smallest eigenvalue of $\hat{R}_{\bar{y}}$.

- Next step, form the matrix $\widehat{\Psi}$.

- Next step, find $\hat{Q}_{o}$ which is a matrix whose columns are chosen to be orthonormal eigenvectors corresponding to the $r$ smallest eigenvalue of $\widehat{\Psi}$ for which we can use "(8)".

- Last step would be obtain channel estimate $\widehat{H}_{o}^{\prime}=$ $\hat{Q}_{o}^{*} \mathrm{~A}$, where $\mathrm{A}$ is an ambiguity matrix.

\section{SIMULATION RESULT}

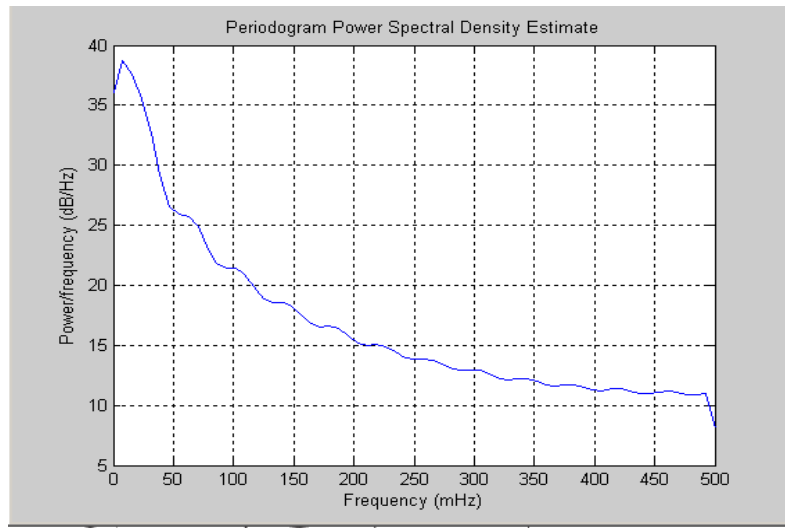

Fig. 4. Power spectral density estimate

Figs. 4 and 5 show the periodogram power spectral density estimation. We are using music algorithm here which estimate the pseudo spectrum from correlation matrix using Schmidt's eigenspace method [20] in order to estimate the signal frequency content. Fig 6 shows the MSE vs. SNR variation, we can observe that our approach is advantageous over previous approach, as the asymptotic performance of [16] does not show advantages over our approach as the eigenvector obtained from sample correlation matrix must lead to less satisfactory estimation performance when time samples are less. In fig. 7 also, the performance is improved using proposed approach. For SNR $\geq 15 \mathrm{~dB}$, we observe that the proposed algorithm employing only 50 OFDM symbols can reach the same performance as if perfect channel-state information (CSI) is known at the receiver.

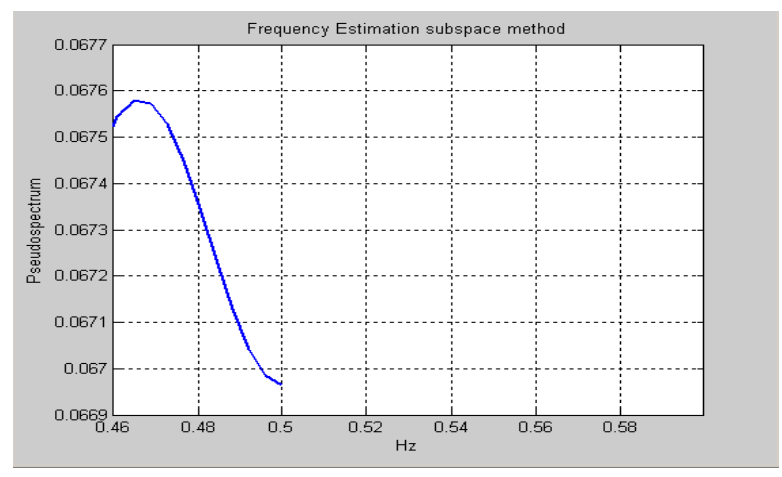

Fig.5. frequency estimation subspace method 


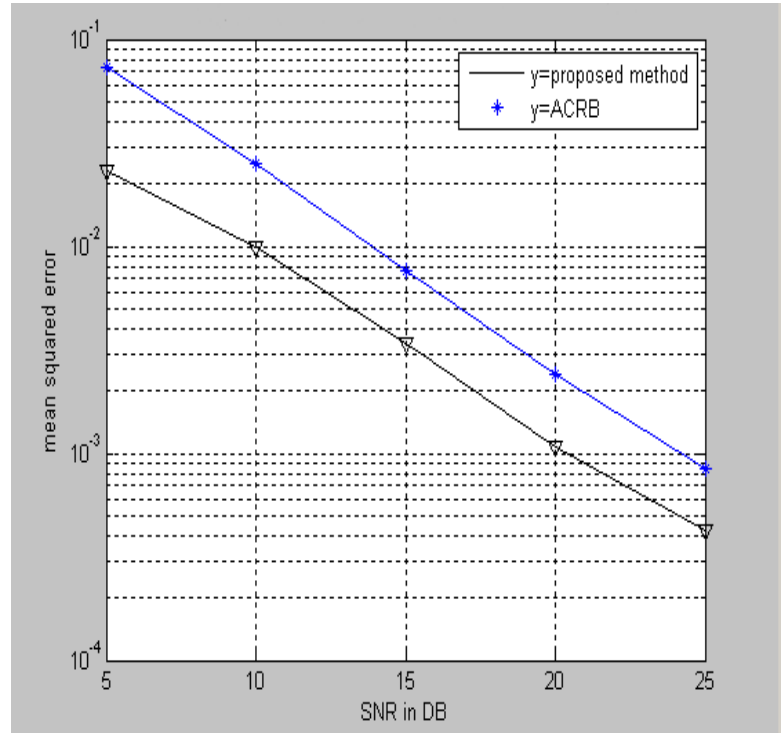

Fig .6. MSE versus SNR for different approach

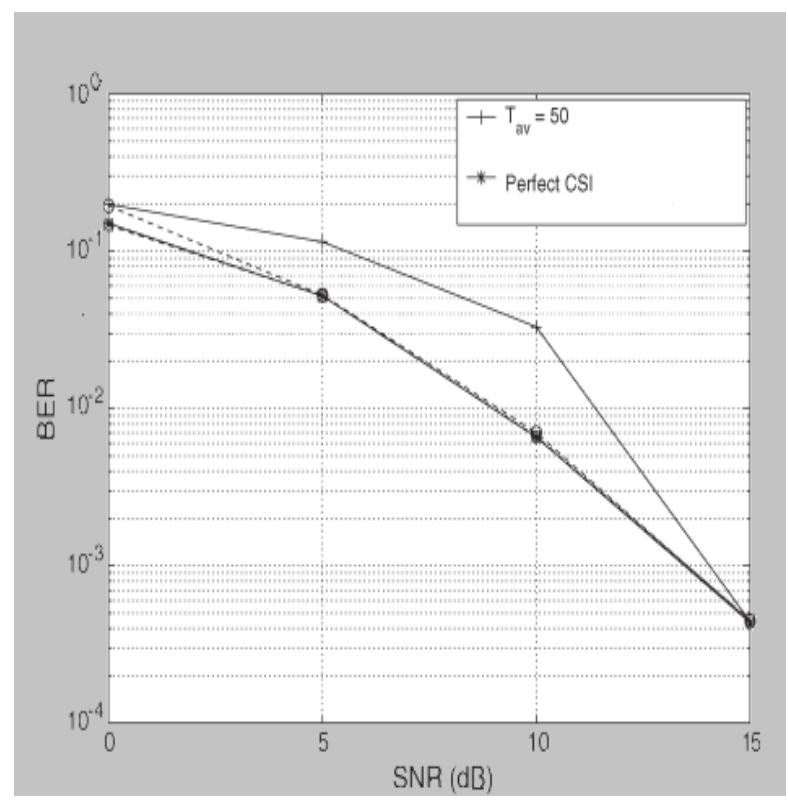

Fig.7. BER versus SNR for proposed approaches

\section{CONCLUSION}

The main contribution of this paper is to improve the performance of MIMO-OFDM system and overcome the limitation of subspace algorithm in time varying channel. The previous subspace method required extremely large number of time samples to obtain a good correlation matrix which make it impractical but in this paper by using frequency correlation among adjacent subcarrier we have prove novel method which require smaller number of time samples. The results of the proposed method have shown that it could achieve a better estimation accuracy than other previously proposed methods and also it is compatible with many existing standards and the coming $4 \mathrm{G}$ wireless communication standards as we are using $\mathrm{CP}$ instead of ZP.

In the future by developing this algorithm we can make it more and more feasible in time varying channel environment, as the continuous variation in channel makes it very difficult to find the channel parameter and estimate the channel. Not only this but the proposed approach can be directly used without any modification of transmitter structure to existing system such as OFDM, SC-CP, etc.

\section{REFERENCES}

[1] R. A. Kennedy and Z. Ding, "Blind Adaptive Equalizers for QAM Communication Systems Based on Convex Cost Functions", SPIE Optical Engineering. June 1992.

[2] L.Tong, G.Xu and T.Kailath "A new approach to blind identification and equalization of multipath channel.," Proc. Aailomar Conf. Signal, Systems and computers, November 1991.

[3] J. A. Cadzow, "Blind deconvolution via cumulant extrema:' IEEE Signal Processing Magazine, vol. 13, no. 3, pp. 2442.May 1996.

[4] L. Tong, G. Xu, and T. Kailath. "Blind identification and equalization based on second-order statistics: A time domain approach," IEEE Transactions on Information Theory, vol. 40, no. 2, pp. 340-349, Mar. 1994.

[5] G. Xu, H. Liu, L. Tong, and T. Kailath, "A least-squares approach to blind channel identification," IEEE Trans. Signal Process., vol. 43, no. 12, pp. 2982-2993, Dec. 1995.

[6] G. B. Giannakis and C. Tepedelenlioglu, "Direct blind equalizers of multiple FIR channels: A deterministic approach," IEEE Trans. Signal Process., vol. 47, no. 1, pp. 62-74, Jan. 1999.

[7] G. J. Foschini, and M. J. Gans, "On limits of wireless communications in a fading environment when using multiple antennas," Wireless Personal Commun., vol. 6, pp. 311-335, 1998.

[8] E. de Carvalho, and D. Slock, "Blind and semi-blind FIR multi channel estimation: (global) identifiability conditions," IEEE Trans. [9]Signal Processing, vol. 52, pp. 1053-1064, Apr. 2004.

[9] K. Abed-Meraim, P. Loubaton, and E. Moulines, "A subspace algorithm for certain blind identification problem," IEEE Trans. Inform. Theory, vol. 32, pp. 499511, Apr. 1997.

[10] C. Shin, R. W. Heath, Jr., and E. J. Powers, "Blind channel estimation for MIMO-OFDM systems," IEEE Trans. Veh. Technol., vol. 56, no. 2, pp. 670-685, Mar. 2007.

[11] TGn Sync Proposal Technical Specification IEEE 802.11-05/1095r2.

[12] Y. Zeng, and T. S. Ng, "A semi-blind channel estimation method for multiuser multiantenna OFDM systems," IEEE Trans. Signal Processing, vol. 52, pp. 1419-1429, May, 2004.

[13] E. Moulines, P. Duhamel, J. F. Cardoso, and S. Mayrargue, "Subspace methods for the blind identification of multichannel FIR filters," IEEE Trans. Signal Processing, vol. 43, pp. 516-525, Feb. 1995.

[14] Y. Li and Z. Ding, "Blind channel identification based on second order cyclostationary statistics," in Proc. IEEE Int. Conf. Acoust., Speech, Signal Process., Apr. 1993, vol. 4, pp. $81-84$.

[15] F. Gao, Y. Zeng, A. Nallanathan, and T.-S. Ng, "Robust subspace blind channel estimation for cyclic prefixed MIMO OFDM systems: Algorithm, identifiability and 
performance analysis," IEEE J. Sel. Areas Commun., vol. 26, no. 2, pp. 378-388, Feb. 2008

[16] L. Tong, G. Xu, and T. Kailath, "Blind identification and equalization based on second-order statistics: A time domain approach," IEEE Trans. Inf. Theory, vol. 40, no. 2, pp. 340-349, Mar. 1994.

[17] L. Tong and S. Perreau, "Multichannel blind identification: From subspace to maximum likelihood methods," Proc. IEEE, vol. 86, no. 10, pp. 1951-1968, Oct. 1998.
[18] L. Tong, G. Xu, B. Hassibi, and T. Kailath, "Blind channel identification based on second-order statistics: A frequency-domain approach," IEEE Trans. Inf. Theory, vol. 41, no. 1, pp. 329-334, Jan. 1995.

[19] http:en.m.wikipedia.org/wiki/eigendecomposition_of_ a_mat-rix.

[20] Schmidt, R.O. "Multiple emitter location and signal parameter estimation." IEEE Transaction on antennas and propagation. Vol. AP-34, March 1986,pp. 276-280. 